\title{
Riscos coletivos e impacto do uso de agrotóxicos sobre a saúde humana e ambiental: um estudo piloto de saúde ocupacional ${ }^{*}$
}

\section{Collective risks and the impact of the use of pesticides about human and environmental health: a pilot study of occupational health}

\author{
Jaime S. LIMAlet \\ Josino C. MOREIRA ${ }^{2}$ \\ Silvana C. JACOB ${ }^{2}$ \\ Alberto J. ARAÚJO ${ }^{3}$ \\ Mônica O. SOARES' \\ Alexandre H. KUBOTA' \\ Marcos C. MONASSA ${ }^{\prime}$ \\ Alexandre M. AMARAL'e \\ Steven MARKOWITZ tes
}

\section{RESUMO}

Este artigo mostra os resultados obtidos em um projeto de avaliação do impacto do uso de agrotóxicos sobre uma comunidade agrícola do estado do Rio de Janeiro ativamente envolvida com o uso de pesticidas. A abordagem utilizada foi de avaliação de riscos ocupacionais e coletivos, já que além do grupo de trabalhadores ocupacionalmente expostos aos agrotóxicos. também foi avaliado um grupo de crianças e jovens da comunidade. alguns dos quais também trabalhadores rurais. Os resultados obtidos demonstram claramente que ocorre superexposição aos pesticidas utilizados. evidenciando agravos não só à saúde das populaçòes expostas, mas também ao meio ambiente.

Palavras-chave: saúde ocupacional, agrotóxicos. organofosforados

\footnotetext{
* Correspondencias devem ser emviadas para: LACAT-Laboratório de Análises Clinicas, Ambientais e Toxicológicas. Universidade do Rio de Janeiro - Unirio. Rua Frei Caneca, 94, 4." andar. Centro. Rio de Janeiro-R.J. CEP 20211-040. O presente traballo foi parcialmente financiado por: a) Fnvironmental and Oceupational Medicine Training Program. Grant ID43 Tw00640 - Fogaty International Center of the National Justitutes of Health: b) Programa de Apoio à Pesquisa - Papes/ Fiocruz: c) Programa de Apoio à Pesquisa e Formaçào de Recursos ma Área de Saude e Segurança no Trabalho. RH - Saúde e Segurança no Trabalho. Prog̣rama Capes/ Findacentro.

1 I. ACAT- Laboratório de Análises Clinicas, Ambientais e Toxicoligicas. Universidade do Rio de Janciro. UNIRIO. Rua lirei Cankéa, 94 -. 4" andar. Centro. Rio de Janciro - RI. CEP $20211-040$

2 Centro de Estudos da Sande do Traballador e Ecologia llumana. ISscola Nacional de Saude Pública (ENSP) e Instituto Nacional de Controle de (aralidade e Salide (INCQS) Fundaçio Oswaldo Cruz (Fiocruz).

3 Programa de Medicina Ocupacional. Hospital Universitário Clementino Fraga Filho. Universidade lederal do Rio de Jánciro. UFR.

4 Emiromental and Occupational Medicine Training Program. Inving Sellikoff Fellowship Program. Mount Sinai School of Medicine/Queens College.

5 Center for the Biology of Natural Systems (CBNS). Queens College. New York.
} 


\section{ABSTRACT}

This paper shows results obtained from a project whose main purpose was the analysis of the impact of pesticides used in an agricultural community of Rio de Janeiro State, actively involved with pesticide use. Major concerns were given to evaluation of occupational and collective risks. Besides occupationally exposed workers' group, children and young of the community were also considered in the analysis. Results clearly show that pesticides overexposure oceur. giving evidence not only injury risks to human health from exposed groups. but also to the environment.

Key-words: occupational health. pesticides. organophosphates

\section{Introdução}

Com o objetivo de aumentar a oferta de alimentos, sua adequação aos padrões de qualidade estabelecidos pelas agroindústrias e redes de distribuição e diminuir as perdas nas lavouras, há muito estão sendo desenvolvidas tecnologias aplicáveis à agricultura, tais como o desenvolvimento de espécies híbridas mais produtivas e resistentes, a utilização em larga escala de fertilizantes e o controle das pragas através do uso de agrotóxicos. Sob um ponto de vista histórico, o emprego dessas tecnologias levou, inicialmente, a um aumento na produtividade de grãos por hectare, entretanto, já se observa um declínio de produção em áreas de cultivo intensivo (PIMENTEL, 1993; PIMENTE1, 1996). Estima-se, por exemplo, que nos Estados Unidos, o uso de agrotóxicos resultou em aumento de sete vezes na produção agrícola por acre desde a década de 30. Apesar disso, relativamente poucos estudos se dedicaram a avaliar satisfatoriamente o custo ambiental e humano relacionado ao uso maciço destes compostos.

Além de seu emprego na agricultura, estas substâncias foram, e ainda são, amplamente utilizadas em campanhas de saúde pública, com o objetivo de erradicação de doenças transmitidas por insetos vetores. Dos inseticidas utilizados para este fim, o representante mais significativo foi o DDT, notadamente utilizado ao longo de décadas em campanhas para erradicação da malária. Apesar da eliminação desta e de outras doenças transmitidas por vetores nos paises desenvolvidos, a expectativa de que o mesmo acontecesse nos países do terceiro mundo não se concretizou até hoje. Ao contrário, em algumas regiões endêmicas, a incidência destas doenças tem aumentado ao longo dos últimos anos, parcialmente devido à seleção de espécies de insetos resistentes ao DDT e outros inseticidas.
Sob uma avaliação global, ao longo dos últimos 40 anos a produção e uso de pesticidas aumentaram drasticamente. Em 1996, o mercado global de pesticidas movimentou aproximadamente 31 bilhões de dólares (AGROW, 1997). O crescimento nas vendas de pesticidas estabilizou-se nos paises desenvolvidos, porém vem crescendo rapidamente nos países em desenvolvimento. Embora somente $25 \%$ sejam consumidos nos paises em desenvolvimento, o uso destes compostos tem crescido marcadamente nos últimos anos, principalmente na África e América Latina (KEIFFER et al., 1997). No mercado latino-americano, o Brasil representa a maior fatia, equivalente a $35 \%$ do total consumido (WHO/UNEP, 1989).

A organização mundial de saúde estima que, no mundo, a exposição a pesticidas é capaz de causar 20.000 mortes anualmente e pelo menos 3 milhões de casos de intoxicação aguda. Outras estimativas sugerem um número muito maior, equivalente a 25 milhões de casos de intoxicação somente nos paises em desenvolvimento (JEYARATNAM, 1990; KOH; JLYARATNAM, 1996). Estes autores argumentam que as estimativas são bastante incompletas, já que, em sua maioria, são feitas apenas com base em internações hospitalares. Estimativas realizadas sobre estas bases tendem a subestimar bastante 0 panorama mundial dos casos de intoxicações, já que excluem os casos menos severos que não chegam ao hospital, casos mal diagnosticados, ou até mesmo casos assintomáticos. Para ressaltar esta idéia há que se considerar as exposições crônicas a baixas e repetidas doses de agentes tóxicos, que causam efeitos muitas vezes mais graves, entretanto, também de difícil diagnóstico.

A maior prevalência de incidentes e acidentes, tanto no Brasil quanto nos diversos outros países do terceiro mundo, resultando freqüentemente em mortes por intoxicação severa, é reflexo e está intrinsecamente relacionada ao perfil socioeconômico e cultural destes países. 
Normalmente, condições de vida que margeiam a miséria levam à potencialização de agravos à saúde causados pela exposição a substâncias tóxicas. Outros fatores agravantes que podem ser constatados estão diretamente relacionados à deficiência de um sistema eficaz de vigilância em saúde e de políticas públicas voltadas para a análise criteriosa da questão, buscando soluções para este, que é de fato um grave problema de saúde pública.

No caso dos trabalhadores rurais, que pertencem a um segmento representativo de populações ocupacionalmente expostas a pesticidas, observam-se problemas que vão desde a falta do uso de equipamentos de proteção individual até o total desconhecimento dos perigos à saúde associados ao uso desses agentes (WHO, 1986; WIIO/ UNI:P, 1989). Além disso, há que se considerar a contaminação ambiental como um problema paralelo, pois não raro, observa-se a contaminação de solos, águas de superfície e subterrâneas e também dos alimentos. Isto provoca efeitos de biomagnificação e bioacumulação, que são responsáveis pela transferência dos resíduos tóxicos ao longo dos diversos níveis tróficos da cadeia alimentar.

A partir da visão geral deste problema, deve-se encarar a necessidade absoluta de programas de avaliação do perfil de saúde dos trabalhadores expostos a agrotóxicos. Deve-se observar que tais programas devem ser integrados, através de alças de retroalimentação, a linhas e projetos de pesquisa científica, programas de vigilância sanitária e outras políticas de saúde pública. Portanto, este aspecto integrador deve ser multidisciplinar e multicêntrico, envolvendo profissionais e instituições de diversas áreas. Desta maneira, as discussões sobre este tema podem ser amplificadas a ponto de permitir e promover um debate mais amplo na sociedade sobre a questão de contaminação ambiental e humana por agrotóxicos.

Para que seja possível esclarecer a sistemática das etapas a serem cumpridas em um programa como este, é fundamental a caracterização de alguns preceitos básicos. Em sintese, qualquer programa de saúde ocupacional deve ter dois objetivos básicos: a proteção de trabalhadores expostos a riscos associados ao ambiente de trabalho e a promoção de medidas que visem a manter a saúde destes trabalhadores. Para alcançar estes objetivos, quatro atividades básicas devem ser consideradas: 1) gerenciamento de risco; 2 ) informação e treinamento; 3) primeiros socorros em situações de emergência, e 4) tratamento médico. Obviamente estas etapas não são necessariamente consecutivas ou mutuamente excludentes, entretanto, o gerenciamento de riscos reveste-se de vital importância e configura-se como a principal atividade no que concerne à proteção à saúde de trabalhadores expostos. O objetivo principal desta atividade é reduzir os riscos a níveis insignificantes ou até mesmo negligentes, de maneira que a força de trabalho não sofra os efeitos adversos lesivos à saúde provocados pelo trabalho ou o ambiente de trabalho. Este objetivo somente pode ser alcançado através de uma abordagem multidisciplinar, envolvendo especialistas da área de saúde e da segurança do trabalho.

Em saúde ocupacional, o termo gerenciamento de risco tem sido usado para descrever o processo como um todo, que inclui uma etapa essencial denominada avaliação de risco. Esta é entendida atualmente como a etapa que inclui as seguintes atividades básicas: identificação de risco, avaliação de exposição e caracterização do risco. As outras atividades que compõem o processo de gerenciamento de risco incluem medidas de controle, informação, instrução e treinamento e vigilância sanitária.

Para a etapa de identificação de riscos à saúde, em programas de avaliação ocupacional relacionados à exposição a agrotóxicos, é necessário ter acesso ao maior número de informações existentes sobre as substâncias utilizadas. Para isto, deve-se identificar e inventariar os produtos aos quais os individuos estão expostos, obter informações, de maneira geral, relacionadas aos dados toxicológicos destas substâncias. Estas informações estão presentes em bancos de dados e edições especializadas, sejam elas nacionais ou internacionais, editadas por órgãos normalmente ligados às áreas de trabalho, saúde e meio ambiente. Também neste processo serão importantes as informações fornecidas pelo fabricante, tais como as monografias para a concessão de autorização legal de produção e venda e, principalmente, aquelas presentes na rotulagem dos produtos. Tais produtos são formulações que constituem um princípio ativo e um solvente carreador ou outro ingrediente, que podem ser inertes ou intrinsecamente tão ou mais tóxicos que o princípio ativo, portanto, a compilação de dados sobre a toxicidade tanto dos princípios ativos quanto de outros ingredientes da formulação torna-se um dos mais importantes aspectos da identificação do risco, e auxiliará diretamente na sua caracterização.

O processo de avaliação de exposição propriamente dito será um conjunto de análises qualitativas e quanti- 
LIMA, J. S. et al. Riscos coletiros e impacto do uso de agrotóxicos sobre a sande...

tativas, abordando três aspectos básicos: a análise dos procedimentos e normas de trabalho, o monitoramento do ambiente de trabalho e o monitoramento biológico dos trabalhadores expostos. Com relação aos trabalhadores rurais, a exposição normalmente se dá sucessiva e simultaneamente a formulações de múltiplos compostos, com uma frequiência regular de aplicação e sob diferentes condições. Uma das características mais presentes no meio rural é que tanto os produtores quanto seus empregados ou meeiros estão regularmente envolvidos com todas as atividades relacionadas à exposição aos pesticidas, tais como, formulação, mistura, aplicação, colheita, descarte de embalagens, tratamento de produtos armazenados e transporte. Daí a necessidade de uma observação acurada dos processos de trabalho. Com relação à investigação destes procedimentos, que estão incluídos na história ocupacional do trabalhador, é importante observar, sempre que possível, se a exposição acontece nas seguintes atividades: 1) mistura da formulação e carregamento das bombas de aplicação; 2) processo de aplicação; 3 ) limpeza do equipamento. Outras importantes informações são obtidas através da avaliação de normas de segurança de trabalho, tais como a checagem da descontaminação de equipamentos de proteção individual e roupas, assim como a investigação de hábitos de higiene pessoal. Normalmente, um questionário bem elaborado e aplicado - o treinamento do entrevistador é etapa crucial desta investigação - auxiliará muito na obtenção destas informações.

Parece um tanto quanto ingênuo tentar investigar um fato que é notório, ou seja, sabe-se que, em geral, nos países subdesenvolvidos, o trabalhador agrícola não segue mínimas normas de segurança; entretanto, este tipo de avaliação quase nunca é sistematizada e utilizada como fonte segura de dados para avaliação de risco. A adoção de metodologias específicas para coleta, sistematização e análise destes dados seria muito mais efetiva com relação aos resultados que seriam alcançados.

Outro importante aspecto da avaliação de exposição refere-se às análises quantitativas propriamente ditas, que são empregadas no monitoramento do ambiente de trabalho e monitoramento biológico. No caso da atividade agrícola, o ambiente de trabalho confunde-se com o ambiente no sentido mais completo que este termo apresenta. Sendo assim, as determinações das concentrações de agrotóxicos têm suas limitações, pois dependem de um desenho de amostragem, coleta, processamento de análise e extrapolação de dados mais com- plexos, quando comparados com um ambiente interno fechado (como uma fábrica, por exemplo), para a consolidação de um padrão de exposição. Apesar disso, vários métodos de monitoramento ambiental podem ser empregados como, por exemplo, análises de amostras ambientais, tais como dos alimentos produzidos na própria lavoura onde as substâncias estão sendo aplicadas e também da água de irrigação ou proveniente de rios e lagos próximos aos sítios de uso de pesticidas ( $A P R I A$ et al., 1994).

No entanto, cabe ressaltar que somente a monitorização do ambiente é insuficiente para se avaliar os riscos a que estão submetidas populações expostas a estes compostos, já que nem sempre é possivel estabelecer associações diretas entre exposição e os efeitos nocivos. De fato, indivíduos expostos à mesma concentração do mesmo xenobiótico podem responder de forma diferente, dadas suas características biológicas intrínsecas, portanto, no caso específico de exposição a agrotóxicos, o monitoramento biológico representa um dos métodos mais adequados para avaliação quantitativa do perfil de exposição.

O monitoramento biológico para avaliação de exposição a pesticidas consiste na determinação do ingrediente ativo ou seus metabolitos, de sistemas biológicos alvo afetados por estas substâncias ou de moléculas cujo aumento, diminuição ou até mesmo a simples presença em um determinado meio biológico refletem um distúrbio metabólico ou fisiológico associado direta ou indiretamente à exposição. Estas análises são feitas em meio biológico (sangue, urina, tecidos adiposos etc.), e representam a carga corpórea das substâncias. A maior vantagem do monitoramento biológico sobre o ambiental está relacionada à informação da dose absorvida através das múltiplas vias de exposição, e conseqüentemente, dá uma boa estimativa da dose total absorvida. Quando se consideram aspectos e conhecimentos sobre a toxicocinética e a toxicodinâmica das substâncias, é possível, através do monitoramento biológico, obter-se informação sobre a dose absorvida em um periodo de tempo definido, que pode ser acumulada no corpo, assim como os sistemas biológicos atingidos. Através do monitoramento biológico, também é possível estimar-se a dose absorvida em função de exposições ocupacionais ou não ocupacionais.

De posse destas informações e, principalmente, pesquisando-se sempre estes métodos para atingir um alto nivel de sensibilidade na deteç̧ão de alterações bio- 
lógicas, será possível prever com precisão quase absoluta o grau de exposição em níveis que ainda não tenham atingido o estágio de desenvolvimento da doença ocupacional. Sabendo-se que a doença somente se estabelecerá como reflexo da perda da homeostasia interna do indivíduo ou deste com o ambiente, pode-se sugerir que as técnicas de monitoramento biológico, desde que utilizadas adequadamente, podem funcionar como ferramentas poderosas na reversão de um quadro de instalação da doença.

A magnitude da caracterização do risco, isto é, a combinação da severidade do efeito adverso com a probabilidade de que este efeito venha a ocorrer, dependerá deste conjunto de dados, associados à análise do grau de exposição. Se houvesse possibilidade de alcançar as melhores condições e parâmetros para concluir esta caracterização, certamente deveriam estar incluidos os seguintes itens: identificação do risco e integração destas informações com os dados reais de exposição (medidos ou estimados), monitoramento ambiental e biológico e análise clínica dos indivíduos expostos. O resultado deste processo será a compreensão de aspectos tanto qualitativos (alterações fisiológicas, identificação de procedimentos inadequados), quanto quantitativos (alta, média ou baixa probabilidade de constatação de efeito adverso). O risco será então estimado e poderá ser definido sob três categorias básicas: risco negligível, aceitável e não aceitável. Caso o risco seja avaliado como sendo pertinente a esta última categoria, deverá ser adotado um conjunto de medidas especificas e aperfeiçoadas de controle da exposição, de maneira a impedir o progresso da deterioração do estado de saúde dos trabalhadores expostos, impedindo assim os processos que resultam no aparecimento da doença de origem ocupacional. Caso o risco venha a ser caracterizado como negligivel ou aceitavelmente baixo, estas medidas não serão necessariamente implementadas, entretanto, esta conclusão deve ser sempre revista através de atividades regulares de avaliação de exposição e de programas de vigilância em saúde.

Os programas de vigilância em saúde consistem de atividades regulares de avaliação do "status" de saúde dos indivíduos expostos através de avaliações médicas (exames físicos e clínicos) e de monitoramento biológico. Esta etapa somente será efetiva e eficiente quando associada e integrada ao processo geral de avaliação e gerenciamento de risco, do mesmo modo, as ações neste programa serão desencadeadas e determinadas pela ava- liação do risco. Ressalte-se que novamente o monitoramento biológico estará incluido nestas atividades, servindo então a duas etapas distintas no processo global.

Em condições ideais, o programa de vigilância pressupõe exames clínicos no momento da admissão no emprego (exames pré-admissíveis) que são anteriores ao início da atividade de risco de exposição ocupacional. Estes exames devem ser compostos de uma análise detalhada do histórico ocupacional relacionado às atividades pregressas, associando-as a possíveis exposições; de análises clínicas e de monitoramento biológico, que também servirão para estabelecer parâmetros de referência individuais, que serão comparados com as mesmas análises após a exposição, construindo então um perfil de avaliação dos efeitos adversos à saúde por ela provocados. Estas avaliações devem ser periódicas e, sempre que alterações significativas forem detectadas, devem ser realizadas intervenções que podem até culminar em afastamento temporário das atividades de risco.

A vigilância em saúde deve também estar acompanhada de diversas outras atividades de investigação epidemiológica, que serão essenciais para a descrição e a busca de soluções para os problemas inerentes ao risco de exposição. A finalização deste processo se dará com medidas de prevenção e controle, constituídas de diversos procedimentos principalmente relacionados à informação. Objetiva-se aí formar agentes de saúde e de segurança de trabalho que saibam lidar com o problema do risco de exposição de maneira hábil e rápida para minimizar riscos. Outro fator importante é criar, reproduzir e distribuir em larga escala fontes de informação que provoquem aumento da percepção dos riscos de exposição e contaminação. Quanto maior for a amplitude de alcance destas informações nas populações expostas, melhor serão os resultados alcançados. Medidas de tratamento e primeiros socorros, assim como ações rápidas frente a uma situação de epidemia também serão importantes, e certamente devem constar de qualquer plano de ação em saúde ocupacional, entretanto, a melhor condição seria não necessitar atingir esta situação limite, pois chegar a ela resulta na observação de que etapas anteriores do gerenciamento de risco podem estar fathando em suas premissas e objetivos iniciais.

No ano de 1998, foi implementado no Rio de Janeiro um projeto de pesquisa multidisciplinar e multicêntrico, envolvendo a Fundação Oswaldo Cruz e três universidades públicas do Rio de Janeiro (UIR.J, UNIRIO e UERJJ), cujo objetivo básico foi realizar um estudo piloto testan- 
LIMA, J. S. et al. Riscos coletivos e impacto do uso de agrotóxicos sobre a saíde...

do uma metodologia de investigação que visava a contemplar todas as etapas de um programa de gerenciamento de riscos como descrito acima, buscando realizar levantamento de fatores socioeconômicos, culturais e biológicos relacionados à contaminação ambiental e humana causada pelo uso de agrotóxicos.

Este projeto foi realizado na região da microbacia do Córrego de São Lourenço, situada entre os municípios de Nova Friburgo e Teresópolis, no estado do Rio de Janeiro, Brasil. Além dos trabalhadores rurais diretamente envolvidos na prática agrícola, foi também avaliado um grupo de crianças e jovens direta ou indiretamente exposto aos agrotóxicos. Esta parte do estudo visava a avaliar o alcance dos riscos de exposição e/ou intoxicação pelos químicos empregados na atividade rural, pois se percebe que os riscos de exposição não são somente associados à atividade laboral mas sim abrangentes à coletividade das famílias expostas.

Este trabalho apresenta os resultados obtidos no desenvolvimento deste estudo, apresentando dados relativos aos riscos e os agravos à saúde que podem advir da exposição de trabalhadores rurais aos agrotóxicos. Também são tecidas considerações sobre os riscos coletivos de exposição e o impacto do uso destas substâncias no meio ambiente.

\section{Metodologia}

\section{Caracterização da população estudada}

Para a realização do projeto, foi elaborado e apresentado para cada participante um termo de consentimento livre e esclarecido segundo a resolução n. 196 do Conselho Nacional de Saúde que trata de procedimentos éticos em pesquisas com seres humanos.

Inicialmente foi realizado um levantamento populacional com a identificação dos 612 residentes da comunidade do Córrego de São Lourenço de Nova Friburgo. Para esta primeira avaliação foi empregado o cadastro da associação de produtores rurais da área. Este cadastro foi utilizado para identificar os agricultores da área (produtores rurais, meeiros, empregados) e suas familias. O critério de inclusão para identificar indivíduos expostos foi bem genérico, ou seja, os indivíduos deveriam ser agricultores ativamente envolvidos na lavoura, seja através de atividades de plantação e co- lheita, assim como de aplicação de agrotóxicos na lavoura. Considera-se realizar um procedimento de amostragem aleatória simples, sendo o tamanho da amostra calculado em 102 indivíduos.

Segundo os critérios adotados para a seleção do grupo a ser avaliado, as crianças foram convidadas a participar do trabalho através da explicação do que seria realizado para suas respectivas famílias. O termo de consentimento livre e esclarecido foi apresentado e assinado pelos responsáveis legais por estas crianças e o trabalho foi então iniciado. De um total de aproximadamente 100 crianças convidadas a participar do estudo, 73 aceitaram, o que revelou uma taxa de resposta de aproximadamente $70 \%$.

\section{Avaliação ocupacional dos individuos expostos a agrotóxicos}

Esta avaliação foi baseada em três variáveis de investigação epidemiológica, clínica e monitoramento biológico e análise laboratorial.

\section{Epidemiológica}

Nesta etapa investigaram-se variáveis demográficas, sociais, econômicas, hábitos e procedimentos de trabalho relacionados ao uso de agrotóxicos. Nesta avaliação o instrumento de coleta de dados foi um questionário denominado: "ficha de investigação epidemiológica".

\section{Clínica}

Nesta etapa foi realizada uma abordagem de investigação clínica profunda, com anamnese dirigida para sinais e sintomas de intoxicação por agrotóxicos e análise por diagnóstico diferencial para eliminar os possiveis erros de interpretação de diagnóstico de intoxicação. Foram utilizados dois tipos de instrumentos de coletas de dados. Um é o "questionário médico", que se caracteriza por uma entrevista que identifica dados sociais e de história de patologias pregressas. Também são feitas perguntas relacionadas ao estado de saúde e sinais e sintomas observados pelo próprio individuo quando este utiliza ou utilizava agrotóxicos nos últimos seis meses. Outro instrumento utilizado foi o questionário de exame clínico, que é uma ficha médica que identifica as principais observações clínicas ao exame físico. 
Monitoramento biológico e análise laboratorial

Neste item foram realizados tanto exames laboratoriais clínicos gerais, como hemograma e análise bioquímica do sangue, quanto os mais específicos, de indicadores biológicos de exposição a agrotóxicos. Neste último grupo de exames foi feita análise de butirilcolinesterase plasmática e de acetilcolinesterase eritrocitária, que são indicadores para inseticidas e acaricidas anticolinesterásicos. Convém ressaltar que estes indicadores são amplamente empregados em estudos como este, apesar de se limitarem a auxiliar no diagnóstico de efeito relativo a duas classes de agrotóxicos: organofosforados (OP) e carbamatos.

Para a dosagem destes indicadores foi empregado o método de Ellman (liLLMAN et al., 1961), modificado por Cunha (CUNIIA, 1996) e o kit de análises I:QM, da companhia EQM Research, Cincinnati - Ohio.

\section{Análise dos dados}

A análise descritiva e quantitativa dos dados foi feita empregando-se o software SPSS 8.0 for windows.

\section{Resultados e discussĩo}

O trabalho foi desenvolvido a partir da aplicação de instrumentos de coletas de dados (questionários de investigação epidemiológica, questionário médico e ficha de exame médico) e avaliação de indicadores biológicos de exposição. Os indicadores biológicos analisados foram as atividades enzimáticas sangüíneas de acetilcolinesterase eritrocitária e butirilcolinesterase plasmática.

Alguns dados demográficos descritivos estão mostrados na tabela 1 .

Pode-se perceber que foi analisado um total de 175 indivíduos, divididos em dois grupos: 102 trabalhadores rurais adultos com mais de 20 anos e 73 crianças e jovens até 20 anos. Das 73 crianças e jovens incluídos no estudo, $70 \%$ trabalhavam diretamente na lavoura, auxiliando seus pais na atividade rural. Somente $30 \%$ não estavam diretamente envolvidos com a atividade laboral. Este grupo era constituido principalmente de crianças até 10 anos, idade a partir da qual as crianças passam a se envolver ativamente na produção agrícola e, conseqüentemente, a se expor diretamente aos químicos utilizados na lavoura.

\section{TABELA I - DADOS DESCRITIVOS DOS GRUPOS POPULACIONAIS ESTUDADOS}

\begin{tabular}{ccc}
\hline & $\begin{array}{c}\text { Traballadores } \\
\text { Rurais adultos }\end{array}$ & $\begin{array}{c}\text { Crianças e Jovens } \\
\text { Meio Rural }\end{array}$ \\
\hline $\begin{array}{c}\text { Número total de } \\
\text { individuos }\end{array}$ & 102 & 73 \\
\hline $\begin{array}{c}\text { Sexo }(\%) \\
\text { Masculino } \\
\text { Feminino }\end{array}$ & 74,5 & 69,7 \\
\hline Idade & 25,5 & 30,3 \\
\hline (Media \pm s.d.) & $34,82 \pm 11,5$ & $13,6 \pm 2,37$ \\
\hline
\end{tabular}

O grupo majoritariamente envolvido era do sexo masculino, porém, deve-se observar que no meio rural o regime de cultivo é basicamente familiar. Isto implica que todos os integrantes da família acabam por se envolver direta ou indiretamente com a exposição aos agrotóxicos. Quase a totalidade das mulheres da comunidade referia que ajudava seus maridos a "puxar a mangueira", ou seja, no momento da aplicação dos agrotóxicos com borrifadores, as mulheres se posicionavam atrás de seus maridos puxando as borrachas de conexão entre o borrifador e o recipiente que estocava a formulação do agrotóxico. Isto tem implicações muito importantes do ponto de vista da exposição, pois, dependendo do tipo e tamanho de partículas aerodispersóides da formulação e do sentido e velocidade dos ventos no momento da aplicação, as mulheres poderiam receber uma carga de exposição no contato do pesticida com a pele maior até mesmo que o homem. Isto tem implicações toxicológicas importantissimas, já que do ponto de vista ocupacional, a principal rota de exposição é a dérmica (WOLFF, 1992).

A tabela 2 apresenta a freqüência das classes de pesticidas utilizadas no meio rural e a caracterização de subclasses por grupos. É interessante notar, que numa região relativamente pequena, onde moram aproximadamente 600 pessoas, 58 diferentes formulações de agrotóxicos estavam sendo utilizadas naquele momento da coleta de dados. Destas formulações, a maior parte era de inseticidas organofosforados $(18,5 \%)$. A tabela 3 amplia a informação da tabela 2 no que concerne à identificação dos agrotóxicos mais utilizados. O inseticida organofosforado metamidofós, considerado altamente tóxico (HAYI:S, 1991), estava sendo usado por $90 \%$ do total de trabalhadores adultos do meio rural. 
Uma das etapas mais importantes no desenvolvimento do trabalho é a investigação epidemiológica. Nela avaliam-se parâmetros demográficos, sociais, econômicos, culturais, e sobretudo comportamentais em relação às práticas de consumo e uso de agrotóxicos no meio rural. Esta etapa normalmente subsidia as etapas posteriores nas tomadas de decisão, ou seja, a partir dos dados obtidos no inquérito epidemiológico é possível identificar alguns fatores de risco a serem investigados e ou controlados. A análise descrita na tabela 4 evidencia as atividades desempenhadas pelos agricultores (adultos, crianças e jovens). Todos estão normalmente engajados em todas as atividades, exceto pelo fato de que crianças e jovens estão menos envolvidos com as atividades de formulação e transporte. É interessante notar este resultado, pois de certa forma evidencia algum tipo de proteção que os membros mais velhos da família têm em relação aos mais jovens. A atividade de formulação é uma das mais perigosas do ponto dos padrões de uso dos agrotóxicos, já que se caracteriza pela preparação das formulações que serão aplicadas na plantação. Nesta preparação são utilizados concentrados altamente tóxi$\cos$ que são preparados por diluição. As tecnologias de diluição são extremamente rudimentares e, muitas vezes a mistura é feita com as próprias mãos, estabelecendo o contato direto do tóxico com a pele dos indivíduos expostos. Esta atividade apresenta um risco severo de intoxicação e o fato de crianças não estarem tão envolvidas quanto adultos pode estar relacionado à proteção anteriormente citada ou a um perfil de educação e compreensão mais elaborado por parte das crianças e jovens, favorecendo maior percepção de riscos por este grupo. Uma menor participação de crianças no transporte nos parece óbvio, já que esta atividade envolve o transporte dos produtos, em caminhões, dos centros produtores para os centros de consumo.

Os resultados apresentados na tabela 5 expressam de maneira clara o risco elevado de exposição/contaminação desta população por agrotóxicos. Normalmente esta população deve receber informações sobre o uso seguro e eficaz destas substâncias nas áreas cultivadas. Estas informações devem ser passadas por técnicos extensionistas de instituições governamentais. Apesar de instituições como a Emater-RJ estarem ativamente empenhadas no trabalho extensivo rural, percebe-se um hiato entre o que é feito e o que é realmente percebido como informação importante para os agricultores. Muitas vezes, determinantes confusos são inseridos pelas próprias indústrias produtoras e empresas comercializadoras dos agroquímicos, pois na competição pela venda de seus produtos ao mercado consumidor (agricultores), acabam por repassar informações sobre padrões de consumo e uso nem sempre corretas. De maneira geral, as respostas levaram a crer que os sistemas de informação sobre o emprego correto e seguro dos agrotóxicos são precários. Isto faz com que a imensa maioria dos indivíduos tenha contato dos pesticidas com a pele, não use proteção adequada para aplicar ou para misturar as formulações, e conseqüentemente, sofra os efeitos de um maior risco e severidade de intoxicação. Segundo os dados apresentados, um total próximo a $40 \%$ dos indivíduos respondeu já ter se sentido mal devido ao uso de pesticidas. Isto talvez explique a quantidade aparentemente contraditória de que $88 \%$ tomem banho logo após a aplicação. Este resultado aparentemente demonstra uma percepção proveniente de vivências individuais e coletivas. Normalmente após a jornada de aplicação, os trabalhadores saem com o corpo totalmente impregnado da substância utilizada. As roupas ficam totalmente molhadas e a pele passa a ser a solução de continuidade da formulação. Caso os trabalhadores não tomem banho logo após o término da jornada, é muito provável que ocorram episódios de intoxicação aguda severa. Vários casos como este foram relatados nas entrevistas, tendo alguns deles resultado em morte por intoxicação aguda. A percepção do risco baseada no medo da morte é um dos fatores que faz com que a grande maioria tenha incorporado este hábito de proteção individual. A proximidade das áreas de cultivo às casas dos indivíduos é outro fator de potencialização de riscos coletivos. Observa-se que o material de trabalho na lavoura se mistura aos utensílios domésticos e roupas impregnadas de agrotóxicos que são lavadas com as roupas da família. Estudos científicos comprovam que resíduos de agrotóxicos que são transportados facilmente para o interior das casas, mesmo quando medidas de segurança são adotadas (LOEWENHERZ et al., 1997). Um quadro como este apresentado acima só confirma a severidade de riscos coletivos no ambiente rural, mesmo quando os indivíduos não são expostos diretamente. 
TABELA 2 - PESTICIDAS UTILIZADOS PELA POPULAÇÃO TRABALHADORA DO MEIO RURAL

\begin{tabular}{ccc}
\hline \multicolumn{3}{c}{ Número total de formulações $=58$} \\
\hline CLASSE & Número de formulaç̃es & \% do total \\
\hline Inseticidas & 22 & 38 \\
\hline Fungicidas & 20 & 34 \\
\hline Herbicidas & 7 & 13 \\
\hline Outros & 9 & 15 \\
\hline Total & 58 & 100 \\
\hline
\end{tabular}

\begin{tabular}{ccc}
\hline Distribuiçao por classe & & \\
\hline Inseticidas & 10 & 18.5 \\
\hline Organofosforados & 7 & 12 \\
\hline Piretroides & 6 & 11 \\
\hline Carbankatos & & \\
\hline Fungicidas & 7 & 12 \\
\hline Inorganicos & 4 & 8 \\
\hline Ftabinitrilas & 3 & 5.5 \\
\hline Benzimidazol & 3 & 5.5 \\
\hline Tiocarbamatos & 3 & 5.5 \\
\hline Ditiocarbamatos & 2 & 3.5 \\
\hline Triazol & 1 & 1.7 \\
\hline Ftalimida & & \\
\hline
\end{tabular}

\begin{tabular}{ccc}
\hline Herbicidas & & \\
\hline Uréias Substituidas & 3 & 5 \\
\hline Triazinas & 2 & 3 \\
\hline Fenoxiacéticos & 1 & 1.7 \\
\hline Bupiridilos & 1 & 1.7 \\
\hline Agentes Biológicos & 1 & 1.7 \\
\hline Antibióticos & 1 & 1.7
\end{tabular}


LIMA, J. S. et al. Riscos coletivos e impacto do uso de agrotóxicos sobre a sańde...

TABELA 3 - SUBSTÂNCIAS MAIS FRI:QÜULNTIEMENTIE UTHLIZADAS PILO GRUPO DE: TRABALHADORIES DO MEIO RURAI.

\begin{tabular}{cc}
\hline COMPOSTO & $\%$ DO TOTAI DI: INDIVÍDUOS \\
\hline Metamidolós & 90 \\
\hline Mancozeb & 69 \\
\hline Paraquat & 58 \\
\hline Clorotalonil & 38 \\
\hline Sulfato de Cobre & 38 \\
\hline Detamatrina & 36
\end{tabular}

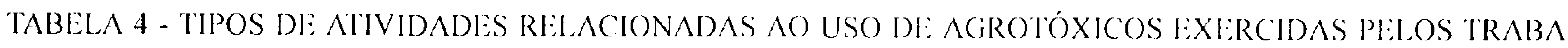
LIIADORES RURAIS I PELAS CRIANÇAS TRABALIIADORAS

\begin{tabular}{|c|c|c|c|c|}
\hline & \multicolumn{2}{|r|}{ SIM } & \multicolumn{2}{|r|}{ NÃO } \\
\hline Atividade & $\%$ de adultos & $\%$ de crianças e jovens & $\%$ de adultos & $\%$ de crianças e jovens \\
\hline Formulaçào & $7 !$ & 33 & 29 & 67 \\
\hline Aplicação & 85 & 75 & 15 & 25 \\
\hline Collheila & 93 & 75 & 7 & 25 \\
\hline Transporte & 55 & 22 & 45 & 78 \\
\hline
\end{tabular}

TABELA 5 - QUIESTÕES RIELATIVAS AO USO DI: AGROTÓXICOS NO MI:IO RURAL,

\begin{tabular}{|c|c|c|c|c|}
\hline & & SIMI & & Nĩo \\
\hline Manuseio com Agrotóxicos & $\%$ de Adultos & $\%$ de Crianças e Jovens & $\%$ de $\wedge$ dultos & $\%$ de Crianças e Jovens \\
\hline Já recebeu algum treinamento? & 48 & 52 & 52 & 48 \\
\hline O extensionista auviliou na forma de usar? & 49 & 29 & 51 & 71 \\
\hline O agrotóxico entra em contato com a pele? & 87 & 78 & 13 & 22 \\
\hline Usa algum tipo de proleção para aplicar? & 35 & 61 & 65 & 39 \\
\hline Usa algum tịo de proteção para misturar? & 13 & 44 & 87 & 56 \\
\hline Toma banho logo após a aplicação? & 88 & 88 & 12 & 12 \\
\hline Passou mal devido ao uso de pesticidas? & 46 & 34 & 54 & 66 \\
\hline
\end{tabular}


É interessante um resultado de frequêencia obtido quando foi realizada a pergunta sobre contato do agrotóxico com a pele. Desta vez as opções de resposta eram: 1) freqüentemente; 2) às vezes e 3) nunca. No grupo de adultos, a freqüência dos que responderam que 0 contato se dava freqüentemente foi de $87 \%$, enquanto no de crianças e jovens foi de $14 \%$ (64\% respondendo às vezes e $22 \%$, nunca). Isto reflete uma diferenciação de práticas de trabalho que pode ter sua origem na educação de crianças e jovens. Quando investiga-se o nivel educacional no grupo, observa-se que $90 \%$ estava freqüentando a escola, sendo que destes, $60 \%$ cursando o ginásio, que no currículo escolar vigente equivale à $2^{\mathrm{a}}$ fase do ensino fundamental ( $5^{n}$ a $8^{a}$ séries). É importante notar que na região existe uma escola alternativa, denominada Instituto Belga, mantida por recursos advindos do governo da Bélgica, na qual as crianças aprendem técnicas agrícolas, além da educação formal. Além disso, o sistema funciona da seguinte maneira: as crianças passam durante todo o periodo letivo por semanas alternadas de ensino e trabalho na terra. Aproximadamente 40 crianças estudavam no Instituto Belga ( $55 \%$ da amostra).

Um outro estudo que merece especial atenção foi a verificação de freqüência de uso de equipamentos de proteção individuais (EPI). Estes equipamentos devem ser utilizados segundo normas de segurança estabelecidas pelo Ministério do Trabalho. Normas de segurança em relação ao uso de agrotóxicos são bem conhecidas e seguem padrões internacionalmente reconhecidos. Apesar disso, a tabela 6 demonstra inequivocamente que, tanto em adultos quanto em crianças, os únicos EPI mais freqüentemente utilizados são botas.

TABILLA 6 - FREQÜIENCIA DI: USO DE EQUIPAMENTO DE PROTEÇÃO INDIVIDUAL DURANTE A APLICAÇÃO DE $\triangle$ GROTÓXICOS NO MEIO RURAL

\begin{tabular}{ccccccc}
\hline & \multicolumn{2}{c}{ Frequie ntemente } & \multicolumn{2}{c}{ Ás vezes } & \multicolumn{2}{c}{ Nunca } \\
\hline E P I & $\begin{array}{c}\% \text { \% de } \\
\text { Adultos }\end{array}$ & $\begin{array}{c}\% \text { de } \\
\text { Crianças e } \\
\text { Jovens }\end{array}$ & $\begin{array}{c}\% \text { de } \\
\text { Adultos }\end{array}$ & $\begin{array}{c}\% \text { de } \\
\text { Crianças c } \\
\text { Jovens }\end{array}$ & $\begin{array}{c}\% \text { de } \\
\text { Adultos }\end{array}$ & $\begin{array}{c}\% \text { de } \\
\text { Crianças e } \\
\text { Jovens }\end{array}$ \\
\hline Náscara de pape I & 4 & 23 & 2 & 13 & 94 & 64 \\
\hline Náscara de bortacha & 6 & 3 & 0 & 4 & 94 & 93 \\
\hline Respirador & 10 & 5 & 4 & 11 & 86 & 84 \\
\hline Luvas & 7 & 27 & 4 & 14 & 81 & 59 \\
\hline Botas & 28 & 54 & 10 & 11 & 62 & 35 \\
\hline Avental & 6 & 7 & 2 & 2 & 92 & 91 \\
\hline Niacacáo & 5 & 9 & 2 & 2 & 93 & 89 \\
\hline Roupas especiais & 4 & 15 & 2 & 5 & 94 & 80
\end{tabular}


$\mathrm{Na}$ realidade, botas são usadas mais para a proteção contra objetos perfuro-cortantes e animais peçonhentos do que propriamente para proteção de exposição a agrotóxicos. Como se pode ver, as práticas de uso de agrotóxicos nesta região só caracterizam um quadro de risco de exposição elevadíssimo, pois observa-se o uso de substâncias de toxicidade elevada associado ao elevado grau de exposição.

O monitoramento biológico realizado neste grupo de indivíduos envolveu a análise de colinesterases como indicadores biológicos de exposição. Estes indicadores são reconhecidamente utilizados em estudos de monitoramento biológico e talvez sejam os indicadores mais utilizados e validados em estudos de monitoramento ocupacional de indivíduos expostos a pesticidas. A limitação de seu uso se dá pelo fato de serem indicadores de efeito somente para as classes de inseticidas organofosforados e carbamatos, pois estas classes de substâncias são conhecidas como agentes anticolinesterásicos, ou seja, agentes com capacidade inibitória sobre as colinesterases.

Sob condições fisiológicas, a acetilcolinesterase hidrolisa a acetilcolina, que é um dos neurotransmissores envolvidos na função sináptica (TAYI.OR, 1991). Na presença de organofosforados, a enzima é fosforilada e, conseqüentemente, acetilcolina acumula nas sinapses nervosas parassimpáticas (ação muscarínica), nas placas terminais motoras (ação nicotínica), e, no sistema nervoso central, esta inibição é responsável por todos os sintomas típicos que ocorrem após envenenamento agudo por organofosforados, os quais podem levar a morte por insuficiência respiratória (RICKI:T et al., 1986; ROSLNBLERY, 1975).

Para a maioria dos organofosforados, no caso de exposição ocupacional, a exposição dérmica e subseqüente absorção através da pele representa a rota de entrada mais importante. As vias oral e respiratória são de menos importância e dependem de circunstâncias especiais. Os efeitos agudos aparecem imediatamente ou logo após a exposição: na exposição dérmica usualmente aparecem sinais e sintomas de intoxicação no período de três a quatro horas após exposição aguda. Um pequeno número de organofosforados pode também reter-se no tecido adiposo, o que provoca uma resposta mais lenta com relação ao aparecimento de tais sinais. A intensidade dos sintomas varia de acordo com o composto (inibidores diretos ou indiretos). Um grande nú- mero destes compostos é rapidamente metabolizado e excretado e, portanto, efeitos subcrônicos e crônicos normalmente não ocorrem. Entretanto, já que estes compostos causam inibição irreversível da acetilcolines-terase, a acumulação deste efeito pode ocorrer. Portanto, sinais e sintomas relacionados a uma única e alta dose podem ser produzidos após pequenas doses repetidas, absorvidas ao longo de uma exposição prolongada.

Existem diferentes tipos de colinesterases no corpo humano, as quais diferem na localização tecidual, afinidades por seus substratos e funções fisiológicas. A principal é a acetilcolinesterase que está no tecido nervoso e eritrócitos. O lento $t u r n o v e r$, eritrocitário faz com que esta enzima possa ser utilizada como um biomarcador de exposição crônica em avaliações de exposição a organofosforados. Também existem as colinesterases plasmáticas, que são produzidas no fígado e continuamente liberadas na corrente sangüinea. Esta atividade também tem sido empregada como um biomarcador de exposição aguda bastante útil para avaliação de exposição a organofosforados (DI:SI et al, 1986; Yl:ARY et al., 1986; NUT1.l:Y, 1993; $\Lambda$ NWAR, 1997). Sabese, também, que existe alta correlação entre a inibição destes sistemas sangüíneos e a inibição que ocorre no sistema nervoso central, o que definitivamente viabiliza - uso destes biomarcadores em programas de monitoramento biológico (PADI1.1^ et al., 1994).

Também deve ser considerado que existem algumas limitações no estabelecimento de índices de desordem funcional, já que existem fatores que provocam variações interindividuais, tais como sexo, idade, polimorfismo genético, gravidez, consumo de álcool, e problemas hepáticos e renais (MUTCH et al., 1992; McQUII:N, 1995; ALI:XIOU et al., 1986), principalmente no que diz respeito a colinesterases plasmáticas. Entretanto, apesar destas variações, estas enzimas têm sido muito úteis como biomarcadores de toxicidade aguda.

Ao longo de décadas as colinesterases têm sido empregadas como os principais indicadores biológicos de exposição a organofosforados, sendo inclusive, desenvolvidas diversas metodologias de campo (kits de diagnóstico) tendo como princípio este indicador de efeito. As normas internacionais que estabelecem limites de exposição ocupacional, ambiental e de tolerância biológica, de maneira geral, indicam que inibições das colinesterases acima de $25 \%$, tomando-se como referência a atividade basal do próprio indivíduo ou de uma 
média de uma população não exposta, já caracterizam niveis de contaminação humana que variam de moderada a grave.

Os niveis de AchE e ChP foram mensurados na população de trabalhadores rurais do Córrego de São Lourenço e, a partir desta análise, pôde-se observar que havia redução dos niveis de colinesterases em aproximadamente $10 \%$ da população estudada.

Para incorporar o monitoramento biológico ao cenário de avaliação de risco, resolve-se desenvolver análise estatística bivariada (Teste $T$ de Student) e multivariada (regressão linear e regressão logística), buscando investigar associações entre a avaliação realizada no monitoramento biológico e as variáveis ocupacionais e clínicas obtidas a partir dos instrumentos específicos (questionários e exames físicos).

No caso dos individuos adultos só foi possível obter associações significativas (valores de $p<0,05$ ) com a acetilcolinesterase eritrocitária. A interpretação que se pôde depreender em relação a esta variável é que foram observados níveis reduzidos da atividade de acetilcolinesterase eritrocitária nos grupos que referiam sintomas como: dor de cabeça, cãibras abdominais, ansiedade, nervosismo e insônia. Também foi observada associação significativa entre o indicador enzimático e fasciculação muscular, que é um sinal observado no exame físico. É interessante notar que estes sinais e sintomas são tipicamente associados à superexposição a organofosforados, e podem estar presentes em quadros de neuropatia induzida por estes compostos.

Modelos de regressão linear múltipla aplicados para investigar associações entre os indicadores biológicos e clínicos evidenciaram valores significativos para os sintomas de dor de cabeça e insônia e associados à diminuição dos niveis de acetilcolinesterase. Quando as variáveis biológicas foram tratadas como variáveis categóricas e colocadas em um modelo de regressão logística, foi possível observar associações significativas entre baixos niveis de acetilcolinesterase e os sintomas de insônia e nervosismo (Odds ratio de 4,85 e 6,22; respectivamente).

$\mathrm{Na}$ análise dos resultados obtidos no estudo com crianças trabalhadoras, todas apresentaram valores normais de acetilcolinesterase, mas cerca de $17 \%$ das crianças avaliadas apresentaram niveis reduzidos de atividade da butirilcolinesterase, o que pode representar uma exposição recente, sem excluir a possibilidade de intoxicação, ou ainda uma distorção nos valores de referência, visto que estes foram obtidos em outra população e para individuos adultos. Na análise por regressão logística, os indices de butirilcolinesterase plasmática foram categorizados como normais e baixos, e muitas variáveis foram inseridas no modelo de regressão, sendo os níveis da enzima plasmática a variável categórica constante. Os resultados que apresentaram associações significativas no modelo de regressão foram: sexo (Odds Ratio $=4,12 ; p<0,05)$ e atividade de aplicação de agrotóxico (Odds Ratio $=5,80 ; p<0,05)$. Estes resultados são importantes, pois, a partir deles pode-se inferir que no grupo investigado as crianças do sexo feminino teriam 4,12 vezes maior probabilidade de apresentar niveis reduzidos de butirilcolinesterase plasmática. Esta constatação é importante principalmente porque as crianças/jovens do sexo feminino ainda se encontram na fase da adolescência, portanto, não há como se prever o impacto que esta exposição poderia trazer para a vida futura das mesmas e de suas familias. Também observa-se que as crianças que trabalham na lavoura, na atividade de aplicação dos agrotóxicos têm 5,80 vezes maior expectativa de apresentar níveis reduzidos de colinesterases. Certamente esta observação mostra, de maneira clara, a associação entre a atividade de aplicação e a diminuição da atividade enzimática, fato este que pode ser determinante para os agravos à saúde decorrentes da exposição.

Outros estudos feitos na mesma região comprovam de maneira inequivoca que ocorre contaminação ambiental significativa. Foi comprovada contaminação da água em diferentes pontos de coleta do rio São Lourenço. Este dado se apresenta revelador se for considerado que não há sistemas de abastecimento de água potável e nem rede de esgoto na região. Toda a população circunvizinha se abastece de água coletada em minas que nascem nas proximidades deste rio. O mesmo estudo identificou contaminação por resíduos de inseticidas organofosforados em elevada percentagem de amostras de tomates, vagem e pimentão provenientes da região e adquiridas no mercado local. Finalmente, um dado muito importante foi a redução da riqueza de táxons na área estudada. Esta redução da diversidade biológica está provavelmente associada a um profundo impacto do uso de pesticidas sobre a biota. Observou-se nesta região um impacto maior principalmente sobre os individuos das ordens Ephemeroptera e Plecoptera, que são organismos reconhecidamente sensíveis (MORI:IRA et al., 2002). 
NUTLEY B. P.: COCKER, J. Biological monitoring of workers occupational exposed to organophosphorus pesticide. Pest. Sci.. [S. 1.]. n. 38, p. 315-322. 1993.

PADILLA. S.: WILSON, V. Z.; BUSHINELL, P. J. Studies on the correlation between blood cholinesterase inhibition and 'target tissue' inhibition insetos pesticide-treated rats. Toxicology. [S. 1.] n. 92. p. 11-25. 1994.

PIMENTEL. D. World Soil Erosion and Conservation. Cambridge: Cambridge University Press. 1993.

PIMENTEL. D. Green revolution agriculture and chemical hazards. The Science of the Total Enviromental. [S. 1.], n. 188 (Suppl.1). p. s86-s98. 1996.

RICKETT, D. L.: GLEEN J. F. E.: BEERS, E. T. Central Respiratory Effects Versus Neuromuscular Actions of Nerve Agents. Neurotoxicology, [S. 1.], n. 7, p. 225-236, 1986.

ROSENBERRY. T. L. Acetylcholinesterase. Adv. Enzimol. Relat. Areas M/ol. Biol. [S. I.], n. 43, p. 103-218, 1975.
TAYLOR, P. The cholinesterases. J. Biol. Chem. [S. I.], v. 266. n. 7. p. $4025-4028,1991$.

WHO. Organophosphorus insecticides: A General Introduction. Environment Heath Criteria, Geneva. n. 63, 1986.

WHO/UNEP. Public Health Impact of Pesticides Used in Agriculture. Geneve: The World llealth Organization/The United Nations Environment Health Program. 1989.

WOLFF, M. S.; MCCONELL, R. Dermal levels of methylparathion, organochlorine pestides and acetylcholinesterase among formulators. Bull. Envron. Contam. Toxicol, [S. 1.|, n. 48, p. 671-678, 1992.

YEARY, R. A.; EATON, J.; GILMORE, E.; NORTH. B.: SINGLE. J. A multiyear study of blood cholinesterase activity in urban pesticides applicators. J. Toxicol. Environ. Health. [S. 1.], n. 39, p. 11-25, 1993. 